\title{
A Phosphate Transporter Gene from the Extra-Radical Mycelium of an Arbuscular Mycorrhizal Fungus Glomus intraradices Is Regulated in Response to Phosphate in the Environment
}

\author{
Ignacio E. Maldonado-Mendoza, Gary R. Dewbre, and Maria J. Harrison \\ The Samuel Roberts Noble Foundation, 2510 Sam Noble Parkway, Ardmore, OK 73401, U.S.A. \\ Submitted 2 April 2001; Accepted 11 June 2001.
}

\begin{abstract}
The majority of vascular flowering plants are able to form symbiotic associations with arbuscular mycorrhizal fungi. These symbioses, termed arbuscular mycorrhizas, are mutually beneficial, and the fungus delivers phosphate to the plant while receiving carbon. In these symbioses, phosphate uptake by the arbuscular mycorrhizal fungus is the first step in the process of phosphate transport to the plant. Previously, we cloned a phosphate transporter gene involved in this process. Here, we analyze the expression and regulation of a phosphate transporter gene (GiPT) in the extra-radical mycelium of the arbuscular mycorrhizal fungus Glomus intraradices during mycorrhizal association with carrot or Medicago truncatula roots. These analyses reveal that GiPT expression is regulated in response to phosphate concentrations in the environment surrounding the extra-radical hyphae and modulated by the overall phosphate status of the mycorrhiza. Phosphate concentrations, typical of those found in the soil solution, result in expression of GiPT. These data imply that $G$. intraradices can perceive phosphate levels in the external environment but also suggest the presence of an internal phosphate sensing mechanism.
\end{abstract}

Arbuscular mycorrhizal (AM) fungi (Zygomycetes: order Glomales) are ubiquitous soil fungi that participate in symbiotic associations with vascular flowering plants in order to develop and complete their life cycle. Their dependency on these associations is the result, at least in part, of a lack of saprotrophic abilities, and these obligate symbionts colonize the cortex of plant roots to obtain carbon from their photoautotrophic partner (Smith and Read 1997). Fossil evidence indicates that arbuscular mycorrhizal fungi have associated with plants for over 400 million years (Remy et al. 1994). Currently, arbuscular mycorrhizas exist in ecosystems throughout the world (Smith and Read 1997).

Corresponding author: M. J. Harrison; Telephone: +1 580-223-5810; Fax: +1 580-221-7380; E-mail: mjharrison@ noble.org

Current address of I. E. Maldonado-Mendoza: CIIDIR-Sinaloa (IPN), Km 1.0 Carretera a Las Glorias, AP280 Guasave, Sinaloa CP 81101, Mexico.

Glomus intraradices phosphate transporter was submitted to GenBank as accession nos. AY037894 and AF359112.
AM fungi have a significant impact on the health and mineral nutrition of their plant symbiotic partner and, although the underlying mechanisms are largely unknown, it is clear that AM fungi can translocate phosphate from the soil to the plant root (Pearson and Jakobsen 1993b; Sanders and Tinker 1971). Phosphate is essential for plant growth and development but is often limiting (Holford 1997), thus access to additional phosphate transported via the AM fungi can have a significant effect on growth and reproduction. Consequently, the AM symbiosis is an important determinant of plant species composition in terrestrial ecosystems (Van der Heijden et al. 1998) and is an essential link for mineral nutrition in sustainable agricultural systems (Jeffries 1987).

At the morphological level, the process of development of the AM symbiosis has been well described (Bonfante-Fasolo 1984; Gianinazzi-Pearson and Gianinazzi 1988). The molecular events underlying the development or nutrient transfer, however, remain to be elucidated (Gianinazzi-Pearson 1996; Harrison 1997). Within the root cortex, the fungus proliferates in the intercellular spaces and differentiates within the cortical cells forming highly branched, terminally differentiated hyphae, called arbuscules. Phosphate transfer between the symbionts is assumed to occur over the arbuscule-cortical cell interface. It is predicted that AM fungi contain an efflux mechanism(s) to release phosphate from the arbuscule and that plants possess a phosphate uptake system to transport phosphate into the cortical cells (Smith 1993; Smith and Smith 1990). A transporter that may mediate phosphate uptake at the arbuscular interface has been identified in tomato (Rosewarne et al. 1999).

In addition to the intra-radical mycelium, the fungus develops an extra-radical mycelium that ramifies in the surrounding soil (Friese and Allen 1991). Radiotracer studies have demonstrated that the extra-radical mycelium is responsible for the acquisition of phosphate, which is later translocated to the intra-radical hyphae and then released to the plant (Jakobsen et al. 1992). As might be anticipated, the obligate symbiotic nature of the AM fungi, coupled with the fact that the symbiosis develops below ground, has posed constraints on the analysis of these fungi and, in particular, access to an intact extra-radical mycelium has been difficult. Consequently, the first kinetic studies of phosphate uptake in AM fungi were performed on hyphal germ tubes (Thomson et al. 1990). These analyses revealed phosphate uptake activities consistent with the pres- 
ence of high- (Km 1.8 to $3.1 \mu \mathrm{M})$ and low-affinity (Km 10.2 to $11.3 \mathrm{mM}$ ) phosphate transport systems, with kinetics similar to those described previously in yeast and Neurospora crassa (Beever and Burns 1977; Tamai et al. 1985). A high-affinity phosphate transporter $(G v P T)$ that shares structural and sequence similarity with the high-affinity, protoncoupled phosphate transporters from yeast (PHO-84) (Bunya et al. 1991) and N. crassa (PHO-5) (Versaw 1995) was later cloned from an AM fungus, Glomus versiforme (Harrison and Van Buuren 1995). GvPT shares $47.9 \%$ identity, at the amino acid level, with the PHO84 from Saccharomyces cerevisiae and $45 \%$ identity, at the amino acid level, with PHO-5 from $N$. crassa. The phosphate transport activity of $G v P T$ was assayed in yeast where it showed a $\mathrm{Km}$ of $18 \mu \mathrm{M}$, a value consistent with its designation as a high-affinity transporter (Harrison and Van Buuren 1995). On the basis of its phosphate transport activity and its expression in the extra-radical mycelium, it is proposed that $G v P T$ plays a role in phosphate acquisition from the soil and is potentially important for phosphate movement in the symbiosis (Harrison and Van Buuren 1995).

Unfortunately, the obligate biotrophic nature of the AM fungi, coupled with the fact that they are asexual, multinucleate organisms (Sanders 1999), makes it impossible to use traditional genetic approaches such as knockout mutants to evaluate the contribution of this transporter to phosphate transport in the symbiosis. Therefore, to further advance our understanding of phosphate transport by AM fungi, we initiated analyses of the expression and regulation of a phosphate transporter gene from Glomus intraradices (GiPT). G. intraradices was chosen for these studies because an in vitro mycorrhiza culture system, that provides access to an intact extra-radical mycelium, has been developed for this species (St. Arnaud et al. 1996). This in vitro system has proved useful for biochemical and cell biology analyses and enabled insight into aspects of metabolism and hyphal architecture (Bago et al. 1998; Pfeffer et al. 1999). Here, we report the analysis of the expression and regulation of the GiPT gene in the extra-radical hyphae of $G$. intraradices during its symbiotic association with plant hosts Medicago truncatula and Daucus carota L. Our data indicate that $G$. intraradices is able to perceive and respond to phosphate levels surrounding the extra-radical mycelium, GiPT is expressed in response to low-phosphate conditions, and the phosphate status of the mycorrhiza influences expression of GiPT.

\section{RESULTS}

\section{Phosphate uptake in M. truncatula-G. intraradices or carrot-G. intraradices mycorrhizas in the in vitro two-compartment plate system.}

In the in vitro culture system developed by St. Arnaud et al. (1996), mycorrhizas are established between transformed carrot roots and $G$. intraradices in one-half of a two-compartment petri dish. As the mycorrhiza develops, extra-radical hyphae proliferate in the medium, surrounding the roots, and eventually grow into the second half of the plate where they further ramify in the medium and later sporulate. To enable manipulation of the environment surrounding the extra-radical hyphae, we modified the system and replaced the solid medium in the second compartment with liquid (Fig. 1). This en- abled manipulation of the environment surrounding the extraradical hyphae and easy access to the hyphae.

The system developed by St. Arnaud et al. (1996) uses Agrobacterium rhizogenes-transformed or "hairy" carrot roots. Transformed roots have the advantage that they grow continuously, as long as they are supplied with carbon. They have an altered sensitivity to phytohormones, however, which might impact the physiology of the mycorrhiza. Therefore, we felt it was important to include experiments with mycorrhizas formed from nontransformed roots. In addition, we wished to evaluate GiPT expression during association of G. intraradices with a second plant host. Consequently, two systems were selected: carrot roots (transformed) and $M$. truncatula roots (nontransformed).

To establish conditions for experimentation, carrot roots or $M$. truncatula roots were placed in the first compartment and inoculated with $G$. intraradices spores. Subsequently, liquid M media, which contains $35 \mu \mathrm{M}$ phosphate, was added to the second compartment. Four weeks later, mycorrhizas had been established and the extra-radical hyphae had grown throughout the media in the first compartment of the plate and into the second compartment of the plate containing the liquid media. At this time, the phosphate content of the liquid media was almost zero, indicating that the fungus had utilized almost all of the available phosphate during this growth period (Fig. 2a). The hyphae remained viable, as determined by nitroblue tetrazolium (NBT)-succinate staining (Schaffer and Peterson 1993). The phosphate content of the M medium surrounding the mycorrhizal roots also was zero at this time (data not shown). The phosphate-depleted liquid media surrounding the extraradical hyphae was then removed and replaced with new media, and the phosphate content of the media was monitored over the subsequent $48 \mathrm{~h}$. In the $M$. truncatula-G. intraradices mycorrhiza and the carrot-G. intraradices mycorrhiza, phosphate was taken up rapidly by the fungal hyphae and $48 \mathrm{~h}$ later, phosphate was no longer detectable (Fig. 2b).

The inclusion of ${ }^{33}[\mathrm{P}]$ orthophosphate in the liquid media confirmed that phosphate was taken up by the extra-radical hyphae and revealed that it was transferred to the mycorrhizal roots in the first compartment of the plate (Table 1). This experiment demonstrates that phosphate is being transported from the external hyphae to the interior of the root, as would be expected for a functional mycorrhiza.

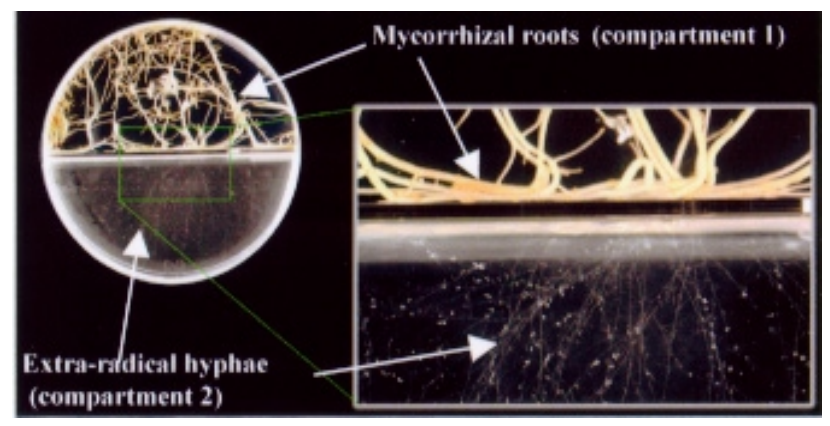

Fig. 1. Photograph of the two-compartment petri dish culture system. Plant roots inoculated with Glomus intraradices spores are cultured in the first compartment. Extra-radical hyphae develop in the second compartment, which contains liquid medium. Arrows indicate mycorrhizal roots and extra-radical hyphae. 
Identification of the $G$. intraradices phosphate transporter (GiPT) gene.

Initial analyses revealed that the $G v P T$ gene hybridized strongly with a transcript from $G$. intraradices. To clone the G. intraradices phosphate transporter gene, a M. truncatulaG. intraradices mycorrhiza cDNA library was screened with a probe corresponding to the coding region of GvPT (U38650). A partial cDNA clone (GiPT) of 1,423 bp was obtained (accession no. AY037894). The GiPT cDNA shares $86 \%$ identity at the nucleotide level and $93 \%$ identity at the amino acid level with $G v P T$. Although the cDNA library contained a high percentage of full-length clones, as determined by analysis of other genes, a full-length GiPT cDNA was not obtained. Subsequently, the full-length GiPT gene was amplified with a primer designed to the $5^{\prime}$ end of the GvPT coding sequence and a $3^{\prime}$ primer based on the GiPT cDNA sequence (accession no. AF359112). The amplified product is $1,665 \mathrm{bp}$ and, in the region of overlap, is $100 \%$ identical with the partial-length GiPT cDNA clone. The coding region of the full-length GiPT shares $90 \%$ identity at the nucleotide level and $95 \%$ identity at the amino acid level with the coding region of GvPT.

\section{GiPT is expressed in the extra-radical mycelium} in response to micromolar levels of phosphate.

A series of two-compartment plates containing developed carrot-G. intraradices mycorrhizas with extra-radical hyphae in the second compartments were established, as outlined above. The phosphate-depleted liquid media surrounding the extra-radical mycelium was then removed and replaced with new media containing $0 \mu \mathrm{M}, 35 \mu \mathrm{M}$, or $3.5 \mathrm{mM}$ phosphate. The extra-radical hyphae were sampled at the time of addition of phosphate and, again, $24 \mathrm{~h}$ later. GiPT transcripts were assayed by ribonuclease protection assay (RPA), and the RNA content of each sample was normalized, relative to an $18 \mathrm{~S}$ rRNA control. This analysis revealed that GiPT transcripts were present in the extra-radical hyphae that received $35 \mu \mathrm{M}$ phosphate but not in the extra-radical hyphae that received $3.5 \mathrm{mM}$ phosphate nor in those that received no phosphate. Transcripts were not detected prior to the addition of phosphate (Fig. 3a). Over this 24-h period, phosphate was taken up by the extra-radical hyphae and, at the time of harvest, the plates that received low $(35 \mu \mathrm{M})$ and high $(3.5 \mathrm{mM})$ phosphate contained $1.2 \mu \mathrm{M}$ and $2.9 \mathrm{mM}$ phosphate, respectively.

Similar GiPT expression patterns were observed in the $M$. truncatula-G. intraradices mycorrhiza. Additional analysis of GiPT transcript levels at intervals following the addition of $35 \mu \mathrm{M}$ phosphate to the extra-radical hyphae of a $M$. truncatula-G. intraradices mycorrhiza revealed that GiPT transcript levels were elevated at 24 and $48 \mathrm{~h}$ post addition of the phosphate (Fig. 3b). The phosphate content of the media surrounding the extra-radical hyphae decreased over time, indicating that phosphate is being taken up by the fungus. These experiments indicate that the expression of GiPT is influenced by the concentration of phosphate surrounding the extra-radical hyphae and that the gene is expressed in response to micromolar concentrations of phosphate. The similarity in the results
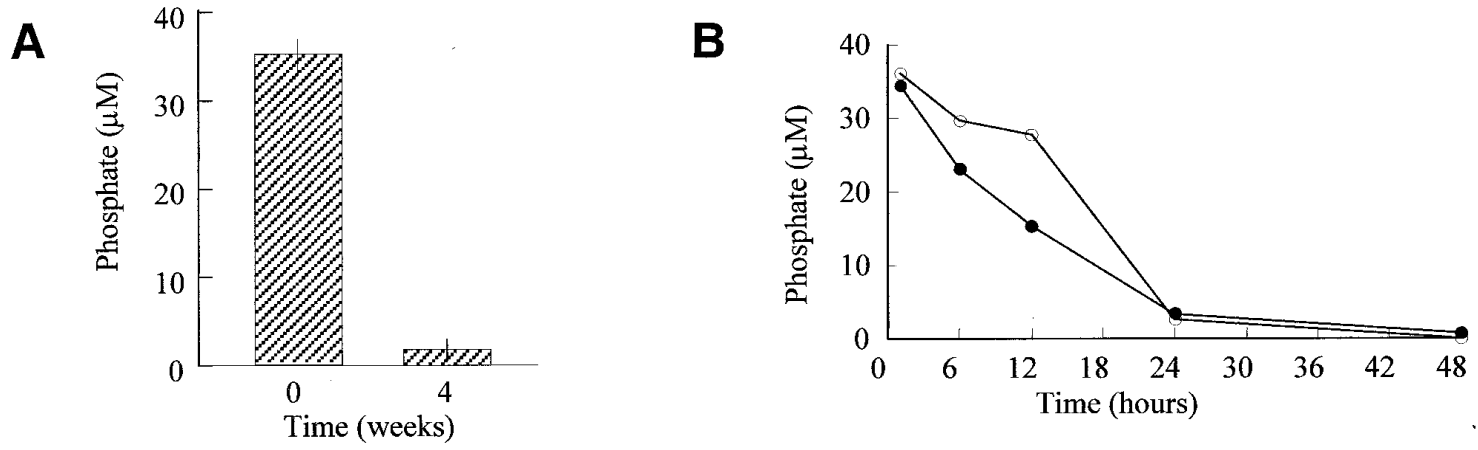

Fig. 2. A, Phosphate concentrations in liquid medium in the second compartment at the time of the addition of M medium (containing phosphate) as well as 4 weeks later when extra-radical hyphae have grown extensively in the medium. Data are derived from three replicates. B, Phosphate concentrations in the media surrounding the extra-radical hyphae following the addition of $35 \mu \mathrm{M}$ phosphate. Phosphate levels in the medium surrounding the extraradical hyphae of a Medicago truncatula-Glomus intraradices mycorrhiza (open circles) and carrot root-G. intraradices mycorrhiza (closed circles).

Table 1. Phosphate translocation from the hyphae to the mycorrhizal roots ${ }^{\mathrm{a}}$

\begin{tabular}{|c|c|c|c|c|}
\hline \multirow{2}{*}{$\begin{array}{l}\text { Time post addition } \\
\text { of phosphate (h) }\end{array}$} & \multirow[b]{2}{*}{${ }^{33} \mathrm{P}$ in liquid medium (\%) } & \multirow{2}{*}{$\begin{array}{c}\text { Phosphate } \\
\text { in liquid medium }(\mu \mathrm{M})\end{array}$} & \multicolumn{2}{|c|}{$\left[{ }^{33} \mathrm{P}\right]$ orthophosphate taken up from liquid medium $(\%)$} \\
\hline & & & In extra-radical hyphae & In mycorrhizal roots \\
\hline 0 & 100 & 35 & & \\
\hline 6 & 82.8 & 28.9 & & \\
\hline 18 & 77 & 26.9 & & \\
\hline 24 & 64.4 & 22.5 & & \\
\hline 30 & 53.3 & 18.6 & & \\
\hline 42 & 25.2 & 8.8 & & \\
\hline 48 & 21.9 & 7.6 & 36 & 10.9 \\
\hline
\end{tabular}

${ }^{a} \mathrm{M}$ media containing $35 \mu \mathrm{M}$ phosphate and $0.5 \mu \mathrm{Ci}\left[{ }^{33} \mathrm{P}\right]$ orthophosphate was added to liquid medium surrounding the extra-radical hyphae of a carrot root-Glomus intraradices mycorrhiza. Liquid medium was sampled over the subsequent $48 \mathrm{~h}$, and the extra-radical hyphae and mycorrhizal roots were sampled at $48 \mathrm{~h}$ post addition of phosphate. Relative amounts of $\left[{ }^{33} \mathrm{P}\right]$ orthophosphate in the medium and tissues are shown. 
obtained with the two plant hosts indicates that this response is not dictated by the plant nor the transformed-nontransformed nature of the roots. Rather, the data support the hypothesis that the fungus has a mechanism via which it can sense phosphate levels and that the GiPT gene is regulated accordingly. As a result of the ease of handling the carrot hairy roots, subsequent experiments were performed with the carrot-G. intraradices arbuscular mycorrhizas.

\section{GiPT transcript levels increase in response to phosphate concentrations typically found in the soil solution.}

The concentration of phosphate in the soil solution is typically $10 \mu \mathrm{M}$ or less (Bieleski 1973) and considerably lower than that used in the initial experiments. To determine whether concentrations of phosphate lower than $35 \mu \mathrm{M}$ would be sufficient to induce GiPT expression, plates were prepared and liquid media containing phosphate at concentrations of 1 to $35 \mu \mathrm{M}$ was placed in the compartment containing the fungal extraradical hyphae. The extra-radical hyphae were harvested at 6 , 12 , and $24 \mathrm{~h}$ following the addition of phosphate, and GiPT transcript levels were assayed by RPA. These assays revealed that GiPT transcript levels do not change in hyphae that receive zero phosphate but are elevated by $6 \mathrm{~h}$ post addition of $1,5,10,20$, or $35 \mu \mathrm{M}$ phosphate (Fig. 4). GiPT transcript levels are clearly higher when the hyphae receive 20 to $35 \mu \mathrm{M}$ phosphate and, consistent with earlier experiments, GiPT transcript levels remain elevated over the next $18 \mathrm{~h}$. On the basis of phosphate concentrations in the media at 6,12 , and $24 \mathrm{~h}$ post addition of phosphate, it is clear that the phosphate is consumed fairly rapidly by the extra-radical hyphae. Consequently, in the plates receiving low phosphate treatments, the phosphate concentration in the media falls to zero very rapidly and elevated GiPT transcript levels are not maintained.

\section{$G i P T$ is regulated in response to changes in phosphate} concentration in the surrounding environment.

To follow the expression of GiPT in the extra-radical hyphae in response to a series of changes in phosphate concentra- tion, plates were prepared and liquid medium containing $35 \mu \mathrm{M}$ phosphate was added to the compartment with the extraradical hyphae. A sample of hyphae was removed $24 \mathrm{~h}$ later and the medium was then replaced with new medium containing $3.5 \mathrm{mM}$ phosphate. After $48 \mathrm{~h}$, a sample of extraradical hyphae was harvested and the hyphae remaining in the plates were washed to remove as much residual phosphate as possible. New medium lacking phosphate was applied. As a result of residual phosphate in the plates, the phosphate concentration of the medium, at this point, was $40.7 \mu \mathrm{M}$ phosphate. A final sample of extra-radical hyphae was removed $48 \mathrm{~h}$ later. RPA analyses revealed that GiPT transcript levels increase dramatically following treatment with low phosphate, then decrease in response to high phosphate, and finally in-

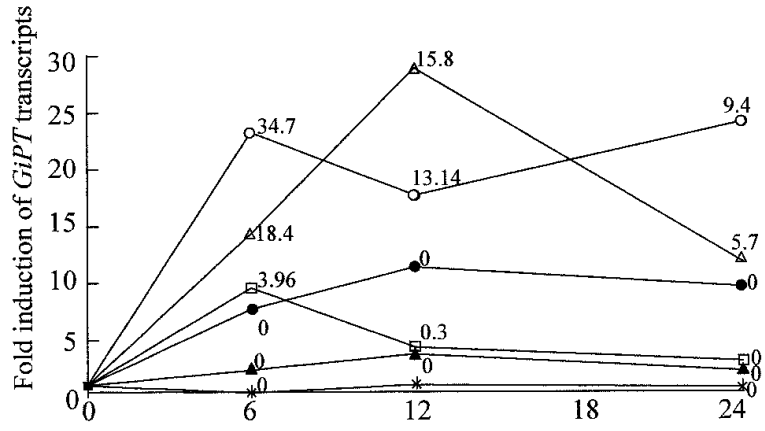

Time (hours)

Fig. 4. Graphical representation of transcript levels of the phosphate transporter gene from Glomus intraradices (GiPT) in extra-radical hyphae of carrot- $G$. intraradices mycorrhizas following exposure to phosphate at concentrations of 1 to $35 \mu \mathrm{M}$. Fold induction is relative to the basal level at time zero, which was set as 1 . Numbers adjacent to the points on the graph indicate the concentration of phosphate in the medium at the time of harvest. Orthophosphate concentrations $(\mu \mathrm{M})$ : asterisks, 0 ; closed triangles, 1 ; closed circles, 5; open squares, 10; open triangles, 20; and open circles, 35.

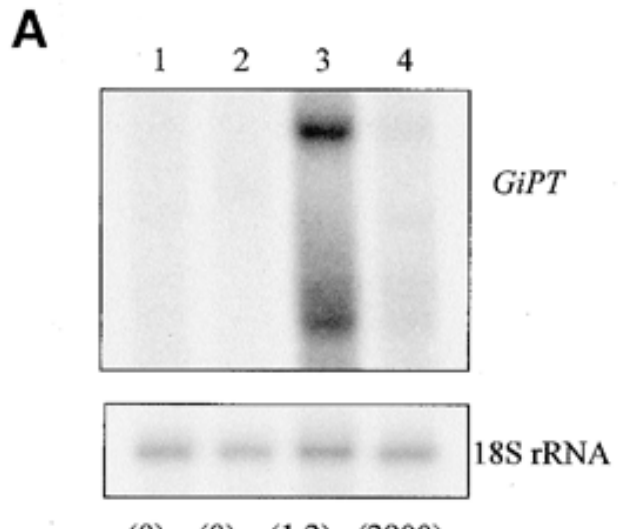

(0) (0) (1.2) (2900)

B

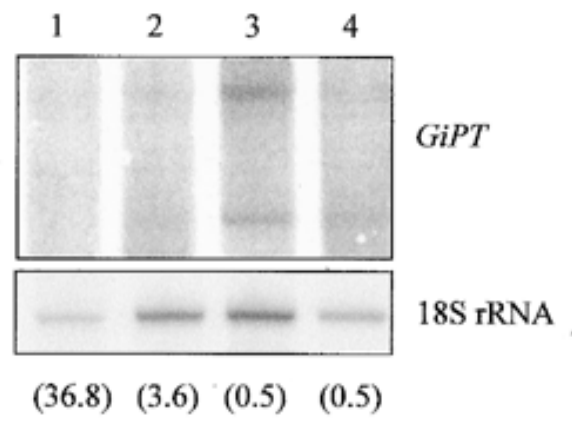

Fig. 3. A, Ribonuclease protection assay detects the phosphate transporter gene from Glomus intraradices (GiPT) transcripts in the extra-radical hyphae of carrot-G. intraradices mycorrhiza prior to (lane 1) and following treatment for $24 \mathrm{~h}$ with $0 \mu \mathrm{M}$ (lane 2), $35 \mu \mathrm{M}$ (lane 3 ), and $3.5 \mathrm{mM}$ (lane 4 ) phosphate. The level of $18 \mathrm{~S}$ rRNA was used to normalize RNA levels in each sample. Numbers in brackets indicate the concentration ( $\mu \mathrm{M}$ ) of phosphate in the medium at the time of harvest. B, Ribonuclease protection assay detects GiPT transcripts in the extra-radical hyphae of a Medicago truncatula-G. intraradices mycorrhiza at 0 (lane 1), 12 (lane 2), 24 (lane 3), and 48 (lane 4) h following treatment with $35 \mu \mathrm{M}$ phosphate. The level of $18 \mathrm{~S}$ rRNA was used to normalize RNA levels in each sample. Numbers in brackets indicate the concentration $(\mu \mathrm{M})$ of phosphate in the medium at the time of harvest. 
crease again as the phosphate concentration decreases (Fig. 5). In a control experiment, high-phosphate conditions were maintained around the extra-radical hyphae and there was no subsequent increase in GiPT transcripts (data not shown).

These data further indicate that GiPT is regulated according to the phosphate conditions surrounding the extra-radical hyphae and support the hypothesis that $G$. intraradices can perceive phosphate in the environment surrounding the extra-radical hyphae.

\section{The phosphate status}

of the mycorrhiza influences expression of GiPT.

To determine whether the phosphate status of the mycorrhiza affects induction of the GiPT gene, plates were prepared and $3.5 \mathrm{mM}$ phosphate was applied to the compartment containing the mycorrhizal roots. The plates were incubated for $48 \mathrm{~h}$, at which point medium containing $35 \mu \mathrm{M}$ phosphate was added to the compartment containing the extra-radical hyphae. Control plates received media containing no phosphate in the mycorrhizal root compartment for $48 \mathrm{~h}$ and then received media with $35 \mu \mathrm{M}$ phosphate in the compartment containing the extra-radical hyphae. The hyphae were sampled over the subsequent $48 \mathrm{~h}$, and GiPT transcript levels were assayed by RPA. In the control plates where the mycorrhizal roots were pretreated with media containing zero phosphate, GiPT transcripts increased in the extra-radical hyphae following the addition of $35 \mu \mathrm{M}$ phosphate, as observed in previous experiments (Fig. 6). GiPT transcript levels increased 21-fold at $3 \mathrm{~h}$ post addition of phosphate and remained at an elevated level of 12- to 16-fold induction for the following $48 \mathrm{~h}$. As in previous experiments, the phosphate content of the media declined and, at $24 \mathrm{~h}$ post addition of phosphate, the phosphate concentration in the media surrounding the hyphae was $6.6 \mu \mathrm{M}$. In the plates where the mycorrhizal roots were pretreated with $3.5 \mathrm{mM}$ phosphate for $48 \mathrm{~h}$, GiPT transcripts

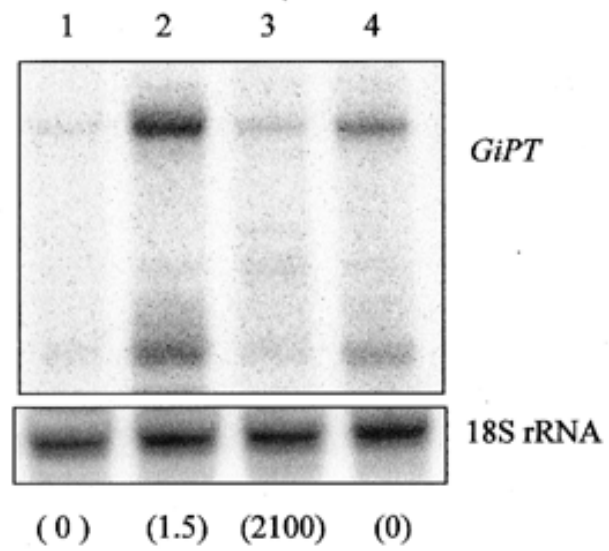

Fig. 5. Ribonuclease protection assay detects transcripts of the phosphate transporter gene from Glomus intraradices (GiPT) in the extraradical hyphae of a carrot- $G$. intraradices mycorrhiza following a series of phosphate treatments. Extra-radical hyphae was sampled (lane 1) and then exposed to $35 \mu \mathrm{M}$ phosphate, and a hyphae sample was removed after $24 \mathrm{~h}$ (lane 2). Remaining hyphae were then exposed to $3.5 \mathrm{mM}$ phosphate, and a second hyphae sample was removed 48 h later (lane 3 ). Finally, the medium surrounding the hyphae was removed and replaced with media containing no phosphate. Residual phosphate from the previous high-phosphate treatment resulted in a phosphate concentration of $40.7 \mu \mathrm{M}$. Hyphae were sampled $48 \mathrm{~h}$ later (lane 4). Values in brackets indicate the phosphate content of the medium at the time of harvest. showed a sixfold induction at $3 \mathrm{~h}$ post addition of phosphate. Transcripts remained at the same level over the subsequent $48 \mathrm{~h}$, and the phosphate levels in the medium surrounding the extraradical hyphae remained approximately $40 \mu \mathrm{M}$. Although the GiPT gene was induced, the fold induction was significantly lower than those plates that did not receive a high phosphate treatment. These data suggest that, if the mycorrhizal roots have sufficient phosphate, then GiPT is not induced to the same levels as seen if the system is deprived of phosphate. Additionally, phosphate is not taken up from the media surrounding the extra-radical hyphae.

\section{GiPT transcript levels increase in response to vanadate.}

In order to obtain information about the nature of the molecule inducing GiPT expression, vanadate, a transition-state analog of phosphate, was tested for its ability to influence expression of GiPT. Plates were prepared, and the compartment containing the extra-radical hyphae was treated with liquid media containing $35 \mu \mathrm{M}$ vanadate or $35 \mu \mathrm{M}$ phosphate. Extraradical hyphae were harvested 24 and $48 \mathrm{~h}$ later. RPAs revealed that GiPT transcripts were not present at the time of the treatment $(0 \mathrm{~h})$ but were detected in the extra-radical hyphae treated with vanadate or phosphate at 24 and $48 \mathrm{~h}$ post addition of these components (Fig. 7). These data indicate that vanadate can induce expression of GiPT to similar levels as phosphate.

\section{DISCUSSION}

Phosphate uptake by AM fungi and its subsequent transport and release to the plant partner in the AM symbiosis is a unique process that has significant impact on plant mineral nutrition and health. Although most plants can obtain phosphate directly from the soil via phosphate transporters in their roots, some studies indicate that in an AM symbiosis, a condition considered the norm in natural environments (GianinazziPearson 1996), the fungal contribution to phosphate uptake by arbuscular mycorrhizal plants may be significantly higher than

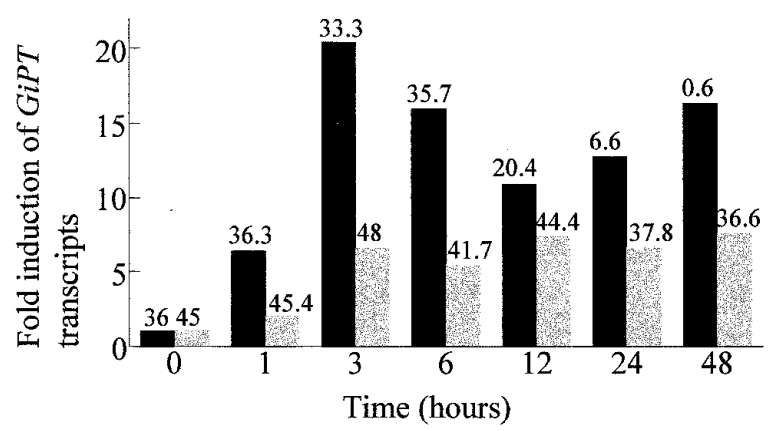

Fig. 6. Graph showing fold induction of transcripts of the phosphate transporter gene from Glomus intraradices $(G i P T)$ in the extra-radical hyphae of carrot-G. intraradices mycorrhizas following pretreatment of the mycorrhizal roots with high phosphate or no phosphate. Mycorrhizas were established, and the first compartment containing the mycorrhizal roots were treated for $48 \mathrm{~h}$ with $\mathrm{M}$ media containing $3.5 \mathrm{mM}$ phosphate or no phosphate. Extra-radical hyphae were then treated with $35 \mu \mathrm{M}$ phosphate, and samples of hyphae were removed over the following $48 \mathrm{~h}$. Black bars represent the fold induction of GiPT transcripts in plates pretreated with no phosphate. Grey bars represent the fold induction of GiPT transcripts in the plates pretreated with high phosphate. Numbers above the bars indicate the phosphate concentration in the medium at the time of harvest. 
that of the plant itself (Pearson and Jakobsen 1993a). The finding that plants down regulate their high-affinity root phosphate transporters in an AM symbiosis is consistent with these studies, and together these data imply that plants may rely heavily on phosphate transport via the fungal symbiont during an AM symbiosis (Chiou et al. 2000; Liu et al. 1998).

As a first step in the analysis of phosphate transport in the $\mathrm{AM}$ symbiosis, we cloned a phosphate transporter gene $(G v P T)$ from the AM fungus G. versiforme (Harrison and Van Buuren 1995). Here, we analyzed the expression and regulation of $G i P T$, a homolog of $G v P T$ from a related fungus, G. intraradices. These studies were facilitated by an in vitro arbuscular mycorrhiza culture system developed for $G$. intraradices (St. Arnaud et al. 1996), which we modified slightly to enable manipulation of the phosphate conditions surrounding the extraradical fungal hyphae and repeated sampling of the hyphae with minimal disturbance to the mycorrhiza. In this system, the mycorrhizas formed between transformed carrot or M. truncatula roots and $G$. intraradices were functional in terms of phosphate transport, and phosphate taken up by the extra-radical hyphae could be detected later in the mycorrhizal roots. Ribonuclease protection assays revealed that, in both mycorrhizal systems, GiPT transcript levels increase in the extra-radical hyphae in response to micromolar levels of phosphate in the surrounding environment. The response is quite rapid and, in some instances, changes in transcript levels could be detected within $3 \mathrm{~h}$. There is some variation between the plates in the magnitude and timing of induction of GiPT and the timing of depletion of phosphate by the extra-radical hyphae. Overall, however, the expression patterns were very consistent. For example, treatment with $35 \mu \mathrm{M}$ phosphate always resulted in a 10- to 30 -fold induction of GiPT transcripts within $24 \mathrm{~h}$ of addition of phosphate. GiPT transcripts were not detected in the absence of phosphate or in response to millimolar levels of phosphate in the surrounding medium. This result also was very reproducible and seen in multiple experiments. These data indicate that the fungus regulates expression of GiPT in response to phosphate conditions surrounding the extra-radical hyphae. Regulation of expression appears to be a dynamic process, as indicated by the increase and decrease in GiPT transcript levels in response to a series of changes in phosphate concentration. These data also imply that $G$. intraradices has a mechanism of sensing phosphate in the environment surrounding the extra-radical hyphae.

The increase in GiPT transcripts in both arbuscular mycorrhizas is accompanied by a decrease in phosphate concentration in the media, which is consistent with phosphate uptake via this transporter. However, as it is not known whether other phosphate transporters are operating in the extra-radical hyphae, it is not possible to say definitively whether this uptake is entirely the result of GiPT. The fact that phosphate is taken up by the external hyphae at high phosphate concentrations when GiPT transcripts are absent (Fig. 5) clearly indicates the presence of another phosphate transport system. Under these conditions, phosphate uptake is probably occurring via a low-affinity phosphate transport system such as the one predicted by Thomson et al. (1990) in Gigaspora margarita, which shows a Km in the order of $10 \mathrm{mM}$. Such transport systems also exist in yeast and $N$. crassa, although the molecular nature of the transport proteins is still unknown (Beever and Burns 1977; Tamai et al. 1985).

Due to the minute amounts of hyphae obtained from this system, it is currently not possible to analyze the GiPT protein levels, and we can only assume that the GiPT transcript levels reflect the protein levels. Previous studies of plant and fungal phosphate transporters suggest that this is a reasonable assumption, and recent studies of the PHO84 transporter indicate that, under high phosphate conditions, the PHO84 protein is removed rapidly from the plasma membrane by endocytosis (Chiou et al. 2000; Lau et al. 2000).

In most experiments, the extra-radical hyphae were initially exposed to $35 \mu \mathrm{M}$ phosphate. Phosphate was not maintained at this concentration, however, and decreased as it was taken up by the extra-radical hyphae. Additional experiments revealed that concentrations of phosphate as low as 1 to $5 \mu \mathrm{M}$ are sufficient to induce expression of GiPT; however, in these cases, phosphate was depleted entirely from media surrounding the hyphae within a few hours and the elevated GiPT transcript levels were not maintained. These data indicate that micromolar levels of phosphate are required not only to induce expression of the GiPT gene but also to maintain expression. This decline in transcript levels also implies that GiPT transcripts turn over relatively quickly. The phosphate concentrations that result in induction of GiPT are consistent with its designation as a high-affinity transporter and with the phosphate concentrations of the soil solution, which are estimated, in most cases, to be less than $10 \mu \mathrm{M}$ (Bieleski 1973).

Based on the expression patterns observed here, GiPT appears to be expressed in a similar manner to the high-affinity phosphate transporters from yeast and $N$. crassa. The yeast PHO84 and N. crassa PHO5 genes are derepressed in response to phosphate-limiting conditions and repressed in response to high-phosphate conditions (Tamai et al. 1985; Versaw 1995). In yeast, the phosphate signaling pathways have been analyzed extensively and PHO84 was found to be under control of the $P H O$ regulatory system (Oshima 1982), with transcription activated by $\mathrm{PHO} 4$ and $\mathrm{PHO} 2$ (Tamai et al. 1985). Under high phosphate conditions, the $\mathrm{PHO} 4$ protein is phosphorylated by a complex formed between a cyclindependant kinase (PHO85) and cyclin (PHO80) (Kaffman et al. 1994). This results in the export of $\mathrm{PHO}_{4}$ to the cytoplasm, thus PHO84 is not transcribed (O'Neill et al. 1996). Under

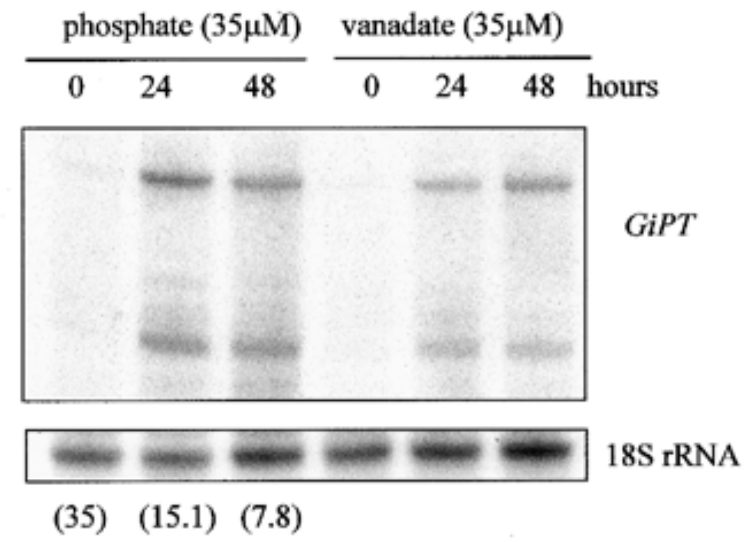

Fig. 7. Ribonuclease protection assay detects transcripts of the phosphate transporter gene from Glomus intraradices (GiPT) in extra-radical hyphae of carrot-G. intraradices mycorrhizas exposed to $35 \mu \mathrm{M}$ phosphate or $35 \mu \mathrm{M}$ vanadate. Extra-radical hyphae was sampled 0, 24, and $48 \mathrm{~h}$ following addition of phosphate or vanadate. Numbers in brackets indicate the phosphate concentration in the medium at the time of harvest. 
low phosphate conditions, a cyclin-dependent kinase (CDK) inhibitor PHO81 inhibits the activity of the PHO85-PHO8O complex and $\mathrm{PHO} 4$ is no longer phosphorylated, remaining in the nucleus where it activates expression of the $\mathrm{PHO} 4 \mathrm{gene}$ (Schneider et al. 1994). A similar system exists in $N$. crassa where the transcriptional activator $N u c l$ is maintained in the cytoplasm during growth in high phosphate conditions and moves to the nucleus under low phosphate conditions (Peleg et al. 1996). Although the mechanisms of regulation of the $G i P T$ gene are entirely unknown, it is feasible that control is mediated via a similar system.

AM fungi not only acquire phosphate for their own use but also release significant quantities to their plant host, a process unique to these fungi. As shown here, GiPT expression is influenced by the phosphate concentration surrounding the extra-radical hyphae and by the phosphate status of the mycorrhizal root system. When the mycorrhizal roots in the first compartment of the petri dish were pretreated with high levels of phosphate prior to the addition of 35 $\mu \mathrm{M}$ to the extra-radical hyphae, GiPT was slightly induced in the extra-radical hyphae but to a lesser extent than the control, which had not received a high phosphate pretreatment. In addition, phosphate was not taken up by the extra-radical hyphae. These data indicate that the phosphate status of the mycorrhizal root influences phosphate uptake by the extra-radical hyphae and GiPT expression. It is not possible to distinguish between an influence from the plant or the fungus because plant and fungal structures in the mycorrhizal roots and extra-radical hyphae surrounding the roots (first compartment) were exposed to high phosphate. In a previous experiment, however, exposure of the extra-radical hyphae to $3.5 \mathrm{mM}$ phosphate did not alter the subsequent expression of GiPT and the gene was still induced in response to low phosphate. Consequently, in this experiment, it seems likely that the phosphate status of the root and/or intra-radical fungal structures impact expression of GiPT. This might occur via a source-sink effect such that phosphate efflux at the arbuscule feedback regulates phosphate uptake in the extra-radical hyphae. Alternatively, a signal from the plant might be involved in regulating GiPT.

How G. intraradices senses phosphate and whether it senses external or internal phosphate concentrations or concentrations of a metabolite such as ATP or polyphosphate is unknown. The finding that vanadate, a transition-state analog of phosphate, also induces expression of GiPT is consistent with the idea that phosphate itself is responsible for the initiation of the signaling cascade that leads to induction of GiPT rather than a downstream metabolite. It is clear that $G$. intraradices regulates GiPT expression in response to external phosphate concentrations. Phosphate sensing, however, could occur via external or internal sensing mechanisms. The observation that GiPT expression is influenced by factors other than the concentration of phosphate surrounding the extra-radical hyphae lends additional support to the existence of an internal phosphate sensing component. The mechanisms of phosphate sensing in fungi are unknown but, taking hints from nutrient sensing in bacteria, internal and external sensing mechanisms are possible.

\section{MATERIALS AND METHODS}

$M$. truncatula-G. intraradices and transformed carrot rootsG. intraradices arbuscular mycorrhizal cultures.

$M$. truncatula seeds were sterilized and germinated as reported previously (Trieu et al. 2000). Excised roots from 3- week-old seedlings were placed on M minimal medium (Bécard and Fortin 1988) containing $20 \mathrm{~g}$ of sucrose per liter in one compartment of a two-compartment petri dish (Fisher Scientific, Pittsburgh, PA, U.S.A.). After 2 weeks, roots were inoculated with $G$. intraradices spores, as described previously (St. Arnaud et al. 1996). At 3 weeks after inoculation, M liquid medium without sucrose was added to the second compartment. Liquid M medium is the same as described by St. Arnaud et al. (1996) but lacks the solidifying agent. The plates were incubated in the dark at $25^{\circ} \mathrm{C}$. It is important not to disturb the plates in order to obtain optimal growth of the extra-radical hyphae. After 4 weeks, extra-radical hyphae developed around the roots in the first compartment and grew into the liquid media in the second compartment. At this point, phosphate was depleted from the liquid and solid media (Fig. 2a) and the mycorrhizas were in a phosphate-depleted state. Plates of this age and phosphate condition were used in all experiments.

The transformed carrot root-G. intraradices arbuscular mycorrhizal system corresponds to the system reported previously by Bécard and Fortin (1988). The carrot hairy root line is DC1. Two-compartment petri dishes containing transformed carrot root-G. intraradices arbuscular mycorrhizas were set up as described above. At 4 weeks after the addition of $\mathrm{M}$ liquid medium to the second compartment, extra-radical hyphae had developed, as described above. At this stage, colonization of the roots was estimated by the modified grid-intersect method (McGonigle et al. 1990) and the average colonized root length was $40.6 \%$, with an average deviation from the mean of $10 \%$.

In the $M$. truncatula and carrot mycorrhizal systems, the roots looked healthy and remained viable throughout the experimental period. Viability was assessed by NBT-succinate staining (Schaffer and Peterson 1993). Approximately 12 weeks after transfer to the plates, the M. truncatula excised roots began to show symptoms of senescence. In contrast, the transformed carrot roots remained viable for a much longer period. All experiments were completed within 10 weeks of transferring the roots to the plates.

\section{Cloning the GiPT.}

$M$. truncatula-G. intraradices mycorrhizas were established in pot cultures, as described previously (Van Buuren et al. 1999). RNA was prepared from $M$. truncatula-G. intraradices mycorrhizal roots at 35 days postinoculation, also as described previously (Harrison and Dixon 1993), and mRNA was prepared from total RNA via an Oligotex mRNA kit (Qiagen, Valencia, CA, U.S.A.). cDNA was prepared with the Uni-ZAP XR synthesis system (Stratagene, La Jolla, CA, U.S.A.) and cloned into the Lambda-ZAP II (Stratagene). The library was screened with a ${ }^{32} \mathrm{P}$-labeled probe corresponding to the coding region of the $G v P T$ gene, according to standard molecular biology procedures (Sambrook et al. 1989). A partial cDNA clone comprising $85 \%$ of the GiPT gene was obtained (GenBank accession no. AY037894).

RNA was prepared from extra-radical hyphae isolated from carrot-G. intraradices mycorrhizas in two-compartment plates with the Purescript RNA isolation kit (Gentra Systems, Minneapolis, MN, U.S.A.). The extra-radical hyphae were treated with $35 \mu \mathrm{M} \mathrm{NaH} \mathrm{PO}_{4}$ for $12 \mathrm{~h}$ prior to harvest to induce the GiPT gene. The GiPT gene was amplified via reverse transcription-polymerase chain reaction (PCR). Total RNA (180 ng) was reverse transcribed with the SuperScript first-strand synthesis system (Life Technologies, Gathersburg, 
MD, U.S.A.). PCR amplification was performed with Taq polymerase (Roche Diagnostics, Mannheim, Germany), with use of an upstream primer corresponding to the $G v P T$ coding sequence $5^{\prime}$-ATGTCTACATCCGATAGAGTAAC- $3^{\prime}$ and a downstream primer based on the GiPT cDNA clone $5^{\prime}$ GGATTTTTATATTCTCCCAATTTATCG-3'. The PCR cycle included 34 cycles, with denaturing at $95^{\circ} \mathrm{C}$ for $30 \mathrm{~s}$, annealing at $50^{\circ} \mathrm{C}$ for $30 \mathrm{~s}$, and extension at $72^{\circ} \mathrm{C}$ for $90 \mathrm{~s}$. The full-length GiPT sequence has been deposited at GenBank as accession no. AF359112.

\section{Transport of $\left[{ }^{33} \mathrm{P}\right]$ orthophosphate in arbuscular mycorrhizas in two-compartment plate systems.}

Phosphate transport in the symbiosis was followed with $\left[{ }^{33} \mathrm{P}\right]$ orthophosphate as a tracer. Plates were established, the media surrounding the extra-radical hyphae was removed, and the hyphae were rinsed three times with $\mathrm{M}$ medium lacking phosphate.

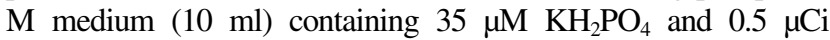
$\left[{ }^{33} \mathrm{P}\right]$ orthophosphate $(3,000 \mathrm{Ci}$ per mmol) (NEN Life Sciences, Boston, MA, U.S.A.) was then added to the extra-radical hyphae. The media surrounding the extra-radical hyphae was sampled over the subsequent $48 \mathrm{~h}$ and analyzed by scintillation counting. The mycorrhizal roots from the first compartment and the extra-radical hyphae were harvested $48 \mathrm{~h}$ after the addition of the $\left[{ }^{33} \mathrm{P}\right]$ orthophosphate. It was not possible to harvest all of the roots because they were embedded in the agar, and approximately $25 \%$ of the roots remained in the plate. The roots and hyphae were immersed in $5 \mathrm{ml}$ of UltimaGold scintillation fluid (Packard Bioscience, Groningen, The Netherlands), and the $\left[{ }^{33} \mathrm{P}\right]$ orthophosphate content was counted in a scintillation counter (Beckman Instruments, Fullerton, CA, U.S.A.).

\section{Manipulation of the phosphate content surrounding} the extra-radical hyphae or around the mycorrhizal roots.

Plates containing extra-radical hyphae in the second compartment were established. The phosphate-depleted medium surrounding the extra-radical hyphae was then removed, and the hyphae rinsed twice with fresh $\mathrm{M}$ medium lacking phosphate. Phosphate treatments were applied to the compartment containing the extra-radical mycelium in $15 \mathrm{ml}$ of $\mathrm{M}$ media. For treatments lacking phosphate, $\mathrm{KH}_{2} \mathrm{PO}_{4}$ was replaced with $\mathrm{K}_{2} \mathrm{SO}_{4}$. In experiments involving a series of treatments, the extra-radical mycelium was washed with $\mathrm{M}$ medium lacking phosphate between treatments. The residual amounts of phosphate after washing were taken into account before proceeding to the next treatment. The extra-radical hyphae were sampled by cutting small sections of hyphae with a small pair of scissors or scalpel and holding the rest of the hyphal network with a fine forceps to avoid separating the hyphal connection to the root system. Care was taken to minimize the disturbance to the system. The phosphate content of the medium was measured before and after the addition of new medium and, at the time of harvest, with the use of a phosphomolybdate colorimetric reaction, in accordance to Chen et al. (1956).

All experiments, except for the $\left[{ }^{33} \mathrm{P}\right]$ orthophosphate (Table 1) and phosphate-vanadate (Fig. 7), were replicated, and single representative data sets are shown. The experiment shown in Figure 3a was repeated five times with the same results.

\section{Ribonuclease protection assays.}

RPAs were performed with the Direct Protect System (Ambion, Austin, TX, U.S.A.) on an aliquot of extra-radical hy- phae. Approximately 5 to $10 \mathrm{mg}$ of extra-radical hyphae could be obtained from a single plate. In a number of experiments, multiple samples were taken from a single plate and, in those cases, the amount of hyphae sampled was $200 \mu \mathrm{g}$ to $1 \mathrm{mg}$. Antisense RNA probes were synthesized by in vitro transcription with the MaxiScript kit (Ambion) and labeled homogeneously with ${ }^{32} \mathrm{P}-\mathrm{UTP}$ (NEN Life Sciences). The G. intraradices phosphate transporter transcripts were detected with an antisense probe corresponding to nucleotides 602 to 1,063 of the $G$. intraradices phosphate transporter gene (accession no. AF359112). To create the probe, an internal fragment from the GiPT gene was amplified with primers (5'-CTG CTG TTG ATT ATT GTT GGC-3' and 5'-GAA CGG TTC CCA TAA TAG TG-3') and cloned into the pGEM-T-easy vector (Promega, Madison, WI, U.S.A.). The plasmid was digested with NcoI, and an antisense probe was created by in vitro transcription from the SP6 promoter. The probe protects a fragment of 461 nucleotides. In the experiment shown in Figure 4, an antisense probe corresponding to the same region of the $G v P T$ gene was used. To create the probe, a 592-bp internal EcoRI-KpnI fragment from the GvPT gene (Harrison and Van Buuren 1995) was cloned into pBluescript $\mathrm{SKII}^{+}$(Stratagene). The plasmid was digested with PstI, and an antisense probe was created by in vitro transcription from the $\mathrm{T}_{7}$ promoter. The probe protects the same nucleotide fragment as the GiPT probe but is three nucleotides shorter (458 instead of $461 \mathrm{bp}$ ). The $G$. versiforme probe shares $86 \%$ identity at the nucleic acid level with the GiPT over this region, and both probes gave identical RPA patterns. Surprisingly, in all of the RPAs, in addition to the 461-bp protected fragment, a second lowermolecular-weight protected band was present. This region of the gene is highly adenine-thymine rich, which can result in weak hybrids that are nicked and, subsequently, digested (Alonso-Prados et al. 1998; Sambrook et al. 1989). The G. intraradices $18 \mathrm{~S}$ rRNA transcript was detected via an $18 \mathrm{~S}$ rRNA probe from $G$. versiforme. The probe protects a fragment of 590 bp (Maldonado-Mendoza et al., in press). The $G$. versiforme and $G$. intraradices $18 \mathrm{~S}$ rRNA sequences are identical in the probe region.

RPAs were carried out according to the manufacturer's instructions (Ambion), except that RNase digestion was performed for 1 $\mathrm{h}$ at $37^{\circ} \mathrm{C}$, and samples were separated by electrophoresis on nondenaturing acrylamide gels, as reported previously (MaldonadoMendoza et al. 1997). To minimize the differences in hybridization efficiencies, the probes were incubated overnight for $16 \mathrm{~h}$ with Direct Protect extracts. The RNase protected bands were quantified by phosphorimager analysis with the Phosphorimager SF (Molecular Dynamics, Sunnyvale, CA, U.S.A).

\section{ACKNOWLEDGMENTS}

The authors thank D. Douds (Penn State University) for the gift of the cultures, Y. Bao (Noble Foundation) and D. Guo (Noble Foundation) for critical reading of the manuscript, and members of the Harrison laboratory for helpful discussions. This work was supported by the Samuel Roberts Noble Foundation.

\section{LITERATURE CITED}

Alonso-Prados, J. L., Aranda, M. A., Malpica, J. M., García-Arenal, F., and Fraile, A. 1998. Satellite RNA of cucumber mosaic cucumovirus spreads epidemically in natural populations of its helper virus. Phytopathology 86:520-524.

Bago, B., Azcón-Aguilar, C., and Piché, Y. 1998. Architecture and devel- 
opmental dynamics of the external mycelium of the arbuscular mycorrhizal fungus Glomus intraradices grown under monoxenic conditions. Mycologia 90:52-62.

Bécard, G., and Fortin, J. A. 1988. Early events of vesicular-arbuscular mycorrhiza formation on Ri T-DNA transformed roots. New Phytol. 108:211-218.

Beever, R. E., and Burns, D. J. 1977. Adaptive changes in phosphate uptake by the fungus Neurospora crassa in response to phosphate supply. J. Bacteriol. 132:520-525.

Bieleski, R. L. 1973. Phosphate pools, phosphate transport and phosphate availability. Ann. Rev. Plant Physiol. 24:225-252.

Bonfante-Fasolo, P. 1984. Anatomy and morphology of VA mycorrhizae. Pages 5-33 in: VA Mycorrhizae. C. L. Powell and D. J. Bagyaraj, eds. CRC Press, Boca Raton, FL, U.S.A.

Bun-ya, M., Nishimura, M., Harashima, S., and Oshima, Y. 1991. The PHO84 gene of Saccharomyces cerevisiae encodes an inorganic phosphate transporter. Mol. Cell. Biol. 11:3229-3238.

Chen, P. S., Toribara, T. Y., and Warner, H. 1956. Microdeterminations of phosphorus. Anal. Biochem. 28:1756-1758.

Chiou, T., Liu, H., and Harrison, M. 2000. The spatial expression patterns of a phosphate transporter (MtPT1) from Medicago truncatula indicate a role in phosphate transport at the root/soil interface. Plant J. 25:1-15.

Friese, C. F., and Allen, M. F. 1991. The spread of VA mycorrhizal fungal hyphae in the soil: Inoculum types and external hyphal architecture. Mycologia 83:409-418.

Gianinazzi-Pearson, V. 1996. Plant cell responses to arbuscular mycorrhiza fungi: Getting to the roots of the symbiosis. Plant Cell 8: 1871-1883.

Gianinazzi-Pearson, V., and Gianinazzi, S. 1988. Morphological integration and functional compatibility between symbionts in vesicular-arbuscular endomycorrhizal associations. Pages 73-84 in: Cell to Cell Signals in Plant, Animal and Microbial Symbiosis. S. Scannerini, D. C. Smith, P. Bonfante-Fasolo, and V. Gianinazzi-Pearson, eds. Springer-Verlag, Berlin.

Harrison, M. J. 1997. The arbuscular mycorrhizal symbiosis: An underground association. Trends Plant Sci. 2:54-56.

Harrison, M. J., and Dixon, R. A. 1993. Isoflavonoid accumulation and expression of defense gene transcripts during the establishment of vesicular-arbuscular mycorrhizal associations in roots of Medicago truncatula. Mol. Plant-Microbe Interact. 6:643-654

Harrison, M. J., and Van Buuren, M. L. 1995. A phosphate transporter from the mycorrhizal fungus Glomus versiforme. Nature 378:626-629.

Holford, I. C. R. 1997. Soil phosphorus: Its measurement, and its uptake by plants. Aust. J. Soil Res. 35:227-239.

Jakobsen, I., Abbott, L. K., and Robson, A. D. 1992. External hyphae of vesicular-arbuscular mycorrhizal fungi associated with Trifolium subterraneum L. 2: Hyphal transport of ${ }^{32} \mathrm{P}$ over defined distances. New Phytol. 120:509-516.

Jeffries, P. 1987. Uses of mycorrhizae in agriculture. Crit. Rev. Biotechnol. 5:319-357.

Kaffman, A., Herskowitz, I., Tjian, R., and O'Shea, E. K. 1994. Phosphorylation of the transcription factor PHO4 by a cyclin-CDK complex, PHO80-PHO85. Science 263:1153-1156.

Lau, W.-T. W., Howson, R. W., Malkus, P., Schekman, R., and O’Shea, E. K. 2000. Pho86p, an endoplasmic reticulum (ER) resident protein in Saccharomyces cerevisiae, is required for ER exit of the high-affinity phosphate transporter Pho84p. Proc. Natl. Acad. Sci. U.S.A. 97:1107-1112.

Liu, H., Trieu, A. T., Blaylock, L. A., and Harrison, M. J. 1998. Cloning and characterization of two phosphate transporters from Medicago truncatula roots: Regulation in response to phosphate and to colonization by arbuscular mycorrhizal (AM) fungi. Mol. Plant-Microbe Interact. 11:14-22.

Maldonado-Mendoza, I. E., Vincent, R. M., and Nessler, C. L. 1997. Molecular characterization of three differentially expressed members of the Camptotheca acuminate 3-hydroxy-3-methyglutaryl CoA reductase (HMGR) gene family. Plant Mol. Biol. 34:781-790.

Maldonado-Mendoza, I. E., Dewbre, G. R., Van Buuren, M. L., and Harrison, M. J. The use of $18 \mathrm{~S}$ probes and ribonuclease protection assays to estimate the proportion of arbuscular mycorrhizal fungal RNA in RNA samples from arbuscular mycorrhizas. In: Proceedings of the 7th International Symposium of the Mycological Society of Japan on Fungus-Plant Interactions. Tsukuba Science City, Japan. In press.

McGonigle, T. P., Miller, M. H., Evans, D. G., Fairchild, G. L., and Swan, J. A. 1990. A new method that gives an objective measure of colonization of roots by vesicular-arbuscular mycorrhizal fungi. New
Phytol. 115:495-501

O'Neill, E. M., Kaffman, A., Jolly, E. R., and O'Shea, E. K. 1996. Regulation of PHO4 nuclear localization by the PHO80-PHO85 cyclinCDK complex. Science 271:209-212.

Oshima, Y. 1982. Regulatory circuits for gene expression: The metabolism of galactose and phosphate. Pages 159-180 in: The Molecular Biology of the Yeast Saccharomyces: Metabolism and Gene Expression J. Strathen, E. Jones, and J. R. Broach, eds. Cold Spring Harbor Laboratory, Cold Spring Harbor, NY, U.S.A.

Pearson, J. N., and Jakobsen, I. 1993a. The relative contribution of hyphae and roots to phosphorus uptake by arbuscular mycorrhizal plants, measured by dual labelling with ${ }^{32} \mathrm{P}$ and ${ }^{33} \mathrm{P}$. New Phytol. 124:489-494.

Pearson, J. N., and Jakobsen, I. 1993b. Symbiotic exchange of carbon and phosphorus between cucumber and three arbuscular mycorrhizal fungi. New Phytol. 124:481-488.

Peleg, Y., Addison, R., Aramayo, R., and Metzenberg, R. L. 1996. Translocation of Neurospora crassa transcription factor NUC-1 into the nucleus is induced by phosphorus limitation. Fungal Genet. Biol. 20:185-191.

Pfeffer, P. E., Douds, J. D. D., Bécard, G., and Shachar-Hill, Y. 1999. Carbon uptake and the metabolism and transport of lipids in an arbuscular mycorrhiza. Plant Physiol. 120:587-598.

Remy, W., Taylor, T. N., Hass, H., and Kerp, H. 1994. Four hundred-million-year-old vesicular arbuscular mycorrhizae. Proc. Natl. Acad. Sci. USA 91:11841-11843.

Rosewarne, G., Barker, S., Smith, S., Smith, F., and Schachtman, D 1999. A Lycopersicon esculentum phosphate transporter (LePT1) involved in phosphorous uptake form a vesicular-arbuscular mycorrhizal fungus. New Phytol. 144:507-516.

Sambrook, J., Fritsch, E. F., and Maniatis, T. 1989. Molecular Cloning: A Laboratory Manual. Cold Spring Harbor Laboratory, Cold Spring Harbor, NY, U.S.A.

Sanders, F. E., and Tinker, P. B. 1971. Mechanism of absorption of phosphate from soil by Endogone mycorrhizas. Nature 233:278-279.

Sanders, I. R. 1999. Evolutionary genetics: No sex please, we're fungi. Nature 399:737-739.

Schaffer, G. F., and Peterson, L. R. 1993. Modifications to clearing methods used in combination with vital staining of roots colonized with vesicular-arbuscular mycorrhizal fungi. Mycorrhiza 4:29-35.

Schneider, K. R., Smith, R. L., and O'Shea, E. K. 1994. Phosphate-regulated inactivation of the kinase PHO80-PHO85 by the CDK inhibitor PHO81. Science 266:122-126.

Smith, S. E. 1993. Transport at the mycorrhizal interface. Mycorrhiza News 5:1-3.

Smith, S. E., and Smith, F. A. 1990. Structure and function of the interfaces in biotrophic symbioses as they relate to nutrient transport. New Phytol. 114:1-38

Smith, S. E., and Read, D. J. 1997. Mycorrhizal Symbiosis. Academic Press, San Diego.

St. Arnaud, M., Hamel, C., Vimard, B., Caron, M., and Fortin, J. A 1996. Enhanced hyphal growth and spore production of the arbuscular mycorrhizal fungus Glomus intraradices in an in vitro system in the absence of host roots. Mycol. Res. 100:328-332.

Tamai, Y., Toh-e, A., and Oshima, Y. 1985. Regulation of inorganic phosphate transport systems in Saccharomyces cerevisiae. J. Bacteriol. 164:964-968.

Thomson, B. D., Clarkson, D. T., and Brain, P. 1990. Kinetics of phosphorus uptake by the germ-tubes of the vesicular-arbuscular mycorrhizal fungus, Gigaspora margarita. New Phytol. 116:647-653.

Trieu, T., Burleigh, S. H., Kardailsky, I. V., Maldonado-Mendoza, I. E., Versaw, W. K., Blaylock, L. A., Shin, H., Chiou, T.-J., Katagi, H., Dewbre, G. R., Weigel, D., and Harrison, M. J. 2000. Transformation of Medicago truncatula via infiltration of seedlings or flowering plants with Agrobacterium. Plant J. 22:531-542.

Van Buuren, M. L., Maldonado-Mendoza, I. E., Trieu, A. T., Blaylock, L. A., and Harrison, M. J. 1999. Novel genes induced during an arbuscular mycorrhizal (AM) symbiosis between Medicago truncatula and Glomus versiforme. Mol. Plant-Microbe Interact. 12:171-181.

Van der Heijden, M. G. A., Klironomos, J. N., Ursic, M., Moutoglis, P., Streitwolf-Engel, R., Boller, T., Wiemken, A., and Sanders, I. R. 1998. Mycorrhizal fungal diversity determines plant biodiversity, ecosystem variability and productivity. Nature 396:69.

Versaw, W. K. 1995. A phosphate-repressible, high-affinity phosphate permease is encoded by the pho- $5^{+}$gene of Neurospora crassa. Gene $153: 135-139$ 